32. McCord, J. M. and Fridovich, I.: Superoxide dismutase: an enzymatic function for erythrocuprein (hemocuprein). J. Biol. Chem., 244: 6049 (1969).

33. Ogawa, J. and Saito, H.: Hyaline membrane in the lung of premature newborn mammals. Study on the etiological factors. Nagoya Med. J., 7: 44 (1961).

34. Paglia, D. E. and Valentine, W. N.: Studies in the quantitative and qualitative characterization of erythrocyte glutathione peroxidase. J. Lab. Clin. Med., 70: 158 (1967).

35. Richards, G. M.: Modifications of the diphenylamine reactions giving increased sensitivity and simplicity in the estimation of DNA. Anal. Biochem., 57: 369 (1974).

36. Rooney, S. A.: Biosynthesis of lung surfactant during fetal and early postnatal development. Trends Biochem. Sci., 4: 189 (1979).

37. Rooney, S. A., Gobran, L. I., Marino, P. A., Maniscalco, W. M., and Gross, I.: Effects of betamethasone on phospholipid content, composition and biosynthesis in the fetal rabbit lung. Biochim. Biophys. Acta, 572:64 (1979).

38. Schacterle, R. E. and Pollack, R. L.: A simplified method for the quantitative assay of small amounts of protein in biological material. Anal. Biochem., 51: 654 (1973).

39. Shanklin, D. R.: On the pulmonary toxicity of oxygen. II. A general theory of oxygen toxicity in man. Perspect. Biol. Med., 13:80 (1969).

40. Thurlbeck, W. M.: Morphologic aspects of bronchopulmonary dysplasia. J.
Pediatr., 95: 842 (1979).

41. Tierney, D. F., Ayers, L., and Kasuyama, R. S.: Altered sensitivity to oxygen toxicity. Am. Rev. Respir. Dis. (suppl.), 115:59 (1977).

42. Wang, N. S., Kotas, R. V., Avery, M. E., and Thurlbeck, W. M.: Accelerated appearance of osmiophilic bodies in fetal lungs following steroid injection. J. Appl. Physiol., 30: 362 (1971).

43. Yam, J., Frank, L., and Roberts, R. J.: Age-related development of pulmonary antioxidant enzymes in the rat. Proc. Soc. Exp. Biol. Med., 157: 293 (1978).

44. Yam, J. and Roberts, R. J.: Pharmacological alteration of oxygen-induced lung toxicity. Toxicol. Appl. Pharmacol., 47: 367 (1979).

45. The authors wish to acknowledge the expert assistance of Ms. Teresa GarciaPons with these studies; and Mrs. Ondina Garcia-Pons with the manuscript preparation. We thank also Dr. Donald Massaro for his multiple contributions.

46. Requests for reprints should be addressed to: Dr. Lee Frank, Pulmonary Division R120, University of Miami School of Medicine, P. O. Box 016960, Miami, FL 33101.

47. Research Supported by NIH Grants No. HL26029 and HL07283.

48. Received for publication December 1, 1982.

49. Accepted for publication June 24, 1983.

\title{
A Comparison of the Sensitivities of Neonatal Ovine Pulmonary and Femoral Arteries to $l$ - Norepinephrine Stimulation
}

\author{
ROBERT S. GREEN ${ }^{(30)}$ AND CHARLES W. LEFFLER
}

Department of Pediatrics, Section of Neonatology-Perinatology, Department of Physiology and Biophysics, The University of Tennessee, Center for the Health Sciences, Memphis, Tennessee, USA

\begin{abstract}
Summary
We compared the concentration-response relationship of intralobar pulmonary arteries to $l$-norepinephrine with that of femoral arteries from newborn lambs. In addition, the effect of inhibition of the neuronal and extra-neuronal uptake mechanisms on these concentration-response relationships was examined.

Concentration-response curves on 10 intralobar pulmonary arteries were performed with and without inhibition of the uptake mechanisms. Uptake inhibition shifts the curve to the left; thus, the $-\log \mathrm{EC}_{50}$ with uptake inhibition $(6.63 \pm 0.15)$ is greater than that without uptake inhibition $(6.13 \pm 0.14)$. Similar curves on 10 femoral arteries demonstrated that the $-\log \mathrm{EC}_{50}$ with and without uptake inhibition $(6.20 \pm 0.13$ and $6.00 \pm 0.04$, respectively) are not statistically different.

Because, in the presence of intact uptake mechanisms the concentration of $l$-norepinephrine in the $\alpha$-receptor microenvironment is less than that placed in the organ bath, the appropriate comparison of pulmonary and femoral arterial response to $l$ norepinephrine stimulation requires uptake inhibition in both vessels. The concentration-response curve with uptake inhibition for the intralobar pulmonary artery is significantly to the left of that for the femoral; the $-\log \mathrm{EC}_{50}$ for the pulmonary vessel $(6.63 \pm 0.15)$ is greater than that of the femoral vessel $(6.20 \pm$ $0.13)$. The intralobar pulmonary artery in vitro is more sensitive to $l$-norepinephrine stimulation than is the femoral.
\end{abstract}

Persistent pulmonary vasoconstriction is a serious, often life threatening problem in the neonatal period $(5,6,21,25,26)$.
There is evidence which suggests that $\alpha$-adrenergic constriction may be involved in the control of the neonatal pulmonary circulation $(4,14)$. Tolazoline, an $\alpha$-adrenergic antagonist, has been used successfully to manage some babies with persistent pulmonary vasoconstriction $(11,17)$ although the therapeutic benefit of this drug also could be related to its histaminergic effects (10). Much remains to be learned about the basic physiology and pharmacology of the newborn pulmonary vascular $\alpha$ adrenergic system. Little is known about the relative sensitivities of various vascular beds in the neonate to $\alpha$-adrenergic stimulation. Differences in sensitivity of different vascular beds to adrenergic agonists such as 1-norepinephrine could have physiologic consequences. As an initial approach to this problem, we compared the concentration-response relationship to 1-norepinephrine of intralobar pulmonary arteries with that of femoral arteries from newborn lambs. In addition, as a prerequisite to this study, the effect of inhibition of the neuronal and extraneuronal uptake mechanisms on these concentration-response relationships was examined. Differences between neonatal pulmonary and femoral arteries were found in both the concentration-response relationship and the effect of inhibition of the uptake systems.

\section{MATERIALS AND METHODS}

Fourteen neonatal lambs (age, 2-8 d; average $4 \mathrm{~d}$ ) were anesthetized with chloralose $(50 \mathrm{mg} / \mathrm{kg}$; Sigma Chemical Co., St. Louis, MO). Femoral arteries were placed in Krebs-bicarbonate buffer; $\mathrm{pH} 7.4$, bubbled with $95 \% \mathrm{O}_{2}-5 \% \mathrm{CO}_{2}$. The left lung was removed, and the pulmonary arterial tree was dissected to the 
apex of each lobe. Fourth generation vessels were cut at their origin, and the intralobar, third generation vessels were removed and placed in Krebs-bicarbonate buffer.

Approximately $2-\mathrm{mm}$ arterial rings were mounted on hooks made from 25 gauge needles (Becton, Dickinson and Co., Rutherford, NJ) and placed in jacketed organ baths (Van Gillum Scientific Glass, Memphis, TN). The bottom attachment of each ring was fixed whereas the top hook was connected to a force transducer with microscale accessory (Statham Model UC-2 transducer from Beckman Instruments, Inc., Schiller Park, IL). Organ bath jackets were circulated with heated water (Haake, Inc., Saddle Brook, NJ) to maintain a bath temperature of $37^{\circ} \mathrm{C}$. The signals from the force transducers were amplified and recorded on a direct writing recorder (R-511A Dynograph Recorder, Beckman Instruments, Inc.).

The length of the rings was adjusted to give an initial baseline tension of $1 \mathrm{~g}$ in the pulmonary vessels and $1.5 \mathrm{~g}$ in the femoral vessels. These baseline tensions were shown in preliminary experiments to be easily definable and to correspond to approximately 1.5 times the unstretched length of each of the two vessel types. This baseline length is near the maximum difference in the active and passive length-tension curves but would not overly stretch the vascular smooth muscle (13). The rings were allowed to reach a steady state $(1-1.5 \mathrm{~h}$ ) with bath changes every $20 \mathrm{~min}$.

Fresh stock solutions of all drugs were made on the morning of an experiment. They were placed in opaque vials and kept on ice. 1-Norepinephrine (Sigma) stocks were made in $10 \mathrm{mM}$ ascorbic acid (Sigma). All experiments were performed in a darkened room to prevent photodecomposition of the catecholamine.

Concentration-response curves were performed with all solutions containing $1 \times 10^{-5} \mathrm{M}, \mathrm{d}, \mathrm{l}$-propranolol (Sigma) to block $\beta$ adrenergic receptors. Concentration-response curves with uptake inhibition were carried out with $3 \times 10^{-5} \mathrm{M}$ cocaine $\mathrm{HCl}$ (Merck \& Co., Inc., Rahway, NJ) and $1 \times 10^{-5} \mathrm{M}$ hydrocortisone (Sigma), inhibitors of neuronal and extra-neuronal uptake respectively $(2,3,12)$. Neither $\beta$-blockade nor uptake inhibition had a significant effect on baseline tension. No more than two curves were done on a vessel in a given day; spontaneous return to baseline was allowed between curves. Order of curves (with and without uptake inhibition) was predetermined so that there would be equal numbers of first curves with and without uptake inhibitors.

1-Norepinephrine concentration-response curves were performed by sequentially increasing the bath concentration from 1 $\times 10^{-8} \mathrm{M}$ to $1 \times 10^{-3} \mathrm{M}$. At each concentration change, the bath was emptied, washed with, and then refilled with a new solution. This washing and refilling procedure caused no change in tension during the baseline equilibration period. The response at each concentration was defined as the change in tension from baseline divided by the maximum change in tension for that vessel. The use of this definition of "response" has been shown to produce 1 -norepinephrine concentration-response curves that are independent of the mechanical baseline conditions (28) and, thus, is useful in comparing the $\alpha$-adrenergic sensitivities of two types of arterial rings.

For each curve reported on a pulmonary vessel under any of the described conditions, a curve was performed simultaneously on a femoral vessel from the same lamb under identical conditions. If, for technical reasons, a curve on an individual vessel could not be completed, the results of its companion vessel were not used. Ten studies on each vessel type with and without uptake inhibition were completed.

Analysis of variance was used for the curves and the paired $t$ test for $-\log \mathrm{EC}_{50} \mathrm{~s}$. The $-\log \mathrm{EC}_{50}$ is the negative value of the logarithm of the norepinephrine concentration expressed in mol/ $\mathrm{L}$ which gives $50 \%$ of the maximum response for a given vessel. The relationship of the $-\log \mathrm{EC}_{50}$ to ring diameter was determined by use of linear regression least-squares analysis with calculation of correlation coefficient. Ring diameters were compared using the paired $t$ test. Values shown represent the mean \pm the standard error of the mean for 10 vessels. All differences cited are significant at $P<0.05$.

\section{RESULTS}

Effect of uptake inhibition. The existence of neuronal (uptake $\mathrm{e}_{\mathrm{I}}$ ) and extra-neuronal (uptake ${ }_{\text {II }}$ ) mechanisms for the removal of 1norepinephrine and other catecholamines from the extracellular space has been documented in several tissues $(12,15,19)$. These uptake mechanisms may lower the concentration of catecholamines in the microenvironment of the $\alpha$-adrenergic receptor; therefore, it has become routine to inhibit these uptake mechanisms when performing 1-norepinephrine concentration-response curves so that the $\alpha_{1}$-receptor is exposed to the same concentration of agonist that exists in the remainder of the organ bath. A necessary preliminary step before examining the relative sensitivities of neonatal pulmonary and systemic vessels is to examine the effect of inhibition of the uptake mechanisms.

Figure 1 shows the results of concentration-response curves on 10 intralobar pulmonary arteries performed with and without inhibition of the uptake mechanisms. Uptake inhibition shifts the curve to the left; thus, the $-\log \mathrm{EC}_{50}$ with uptake inhibition $(6.63 \pm 0.15)$ is greater than that without uptake inhibition $(6.13$ $\pm 0.14)$. The curve with uptake inhibition represents the true position of the concentration-response curve because, in that situation, the $\alpha_{1}$-adrenergic receptor is exposed to the same concentration as is placed in the organ bath.

Figure 2 shows similar results for 10 femoral arteries. The curves with and without uptake inhibition are not different. The $-\log \mathrm{EC}_{50} \mathrm{~s}$ with and without uptake inhibition $(6.20 \pm 0.13$ and $6.00 \pm 0.04$, respectively) are not significantly different. In this experimental model, the uptake mechanisms alter the concentration-response relationship in neonatal intrapulmonary arteries but not in neonatal femoral arteries. Inhibition of the uptake mechanisms did not change the absolute value of the maximum change in tension in either pulmonary arteries $(0.83 \pm 0.13 \mathrm{~g}$ with uptake inhibition and $0.88 \pm 0.13 \mathrm{~g}$ without uptake inhibition) or femoral arteries $(2.5 \pm 0.2 \mathrm{~g}$ with uptake inhibition and $2.8 \pm 0.3 \mathrm{~g}$ without uptake inhibition).

Least-squares analyses of $-\log \mathrm{EC}_{50}$ versus ring diameter yielded correlation coefficients (degrees of freedom $=9$ ) of 0.508 and 0.065 for the femoral and pulmonary rings, respectively, both of which are not significant. Similarly, the shift of the $-\log$ $\mathrm{EC}_{50}$ of the pulmonary arterial rings caused by uptake inhibition

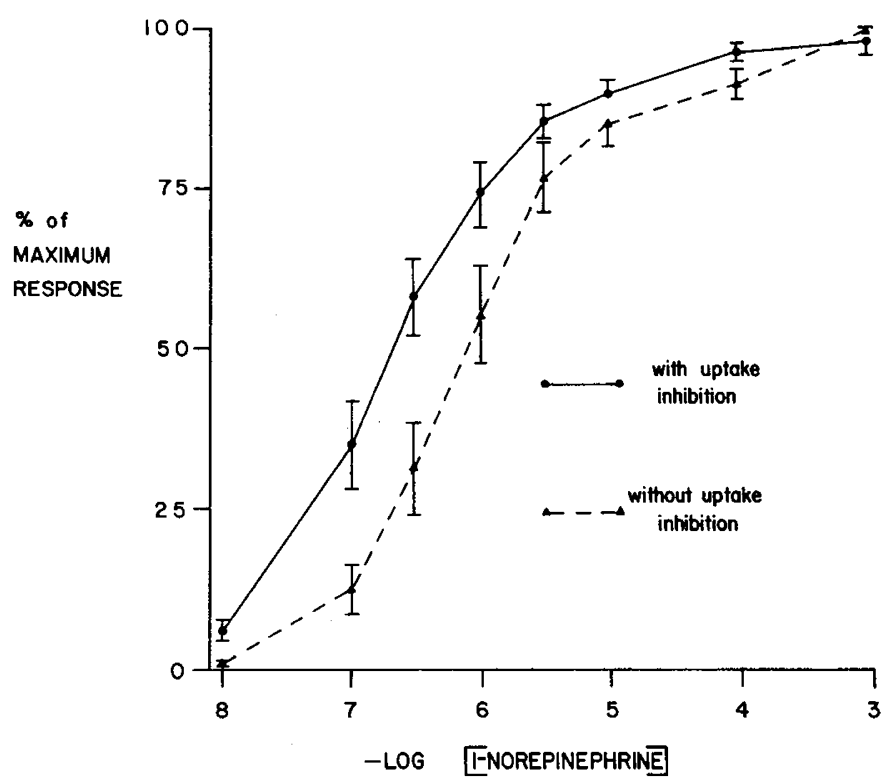

Fig. 1. 1-Norepinephrine (mol/L) concentration-response curves of neonatal ovine intralobar pulmonary arteries $(n=10)$ with and without uptake inhibition. Bars indicate \pm 1 SEM. Curves different $P<0.05$. 


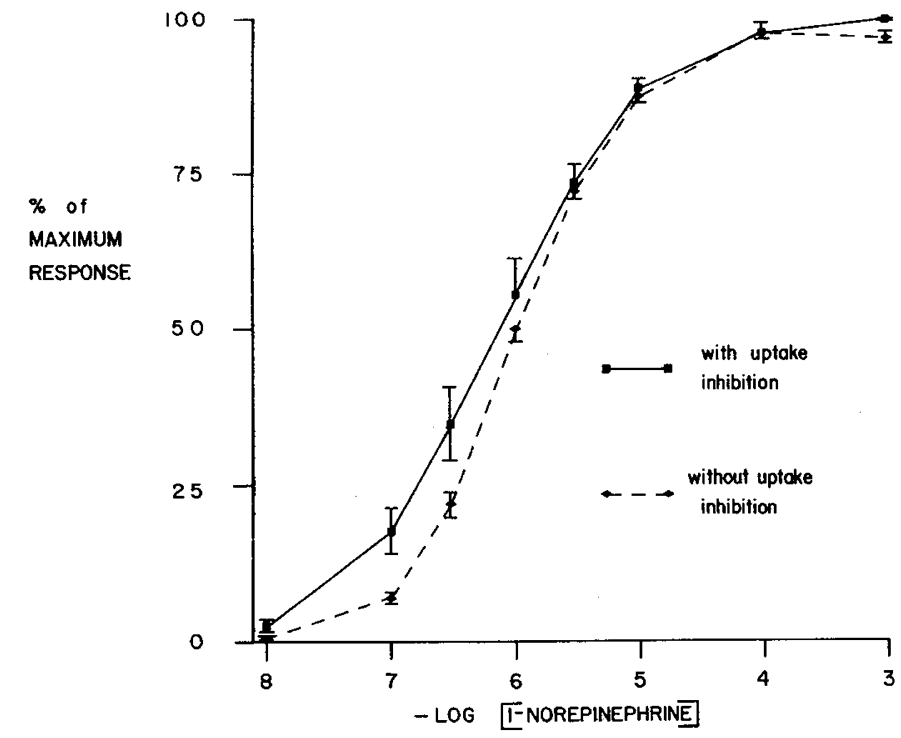

Fig. 2. 1-Norepinephrine (mol/L) concentration-response curves of neonatal ovine femoral arteries $(n=10)$ with and without uptake inhibition. Bars indicate \pm 1 SEM. Curves not significantly different.

was not significantly related to ring diameter (correlation coefficient $=0.310$ ).

Comparison of the pulmonary and femoral responses. The shift of the concentration-response relationship by uptake inhibition in the pulmonary vessels implies that in the absence of the inhibitors, the concentration of 1 -norepinephrine in the $\alpha_{1}$ receptor microenvironment is less than the concentration placed in the organ bath. The appropriate manner in which to compare the response to $\alpha_{1}$-receptor stimulation is to inhibit uptake in both vessels. Figure 3 shows the concentration-response curves of 10 pulmonary vessels and the 10 femoral vessels with uptake inhibition. The concentration-response curve for the intralobar pulmonary artery is significantly to the left of that for the femoral artery; thus, the $-\log \mathrm{EC}_{50}$ for the pulmonary vessel $(6.63 \pm$ $0.15)$ is significantly greater than that for the femoral $(6.20 \pm$ $0.13)$.

Femoral ring diameter $(2.25 \pm 0.16 \mathrm{~mm})$ measured in vitro was less than pulmonary ring diameter $(2.78 \pm 0.17 \mathrm{~mm})$ measured similarly. But, least-squares analysis of the difference in the $-\log \mathrm{EC}_{50}$ of the paired pulmonary and femoral arterial rings versus the difference in their respective diameters showed no significant correlation (correlation coefficient $=0.503$; degrees of freedom $=9$ ).

\section{DISCUSSION}

The potential role for $\alpha$-adrenergic constriction in the control of the pulmonary vasculature is suggested by observations of $\alpha$ adrenergic pulmonary vasoconstriction in a variety of experimental models $(3,4,12,16,27)$. Furthermore, $\alpha$-adrenergic pulmonary vasoconstriction in the neonatal lamb may be altered by hypoxia (20); circulating 1-norepinephrine levels are elevated markedly in stressed human neonates $(18,24)$ and $\alpha$-adrenergic blockade may be useful in treating neonatal pulmonary hypertension $(11,17)$. $\alpha$-Adrenergic stimulation may play an important role in the control of the perinatal pulmonary circulation and in the generation of persistent pulmonary hypertension in the neonate.

In addition, differential effects of vasoactive agents on pulmonary and systemic vessels could be important physiologically in the neonate in whom the pulmonary and systemic circulations are connected by the ductus arteriosus and foramen ovale. As an initial approach to this subject, we compared the concentrationresponse relationship of intralobar pulmonary arteries to 1-

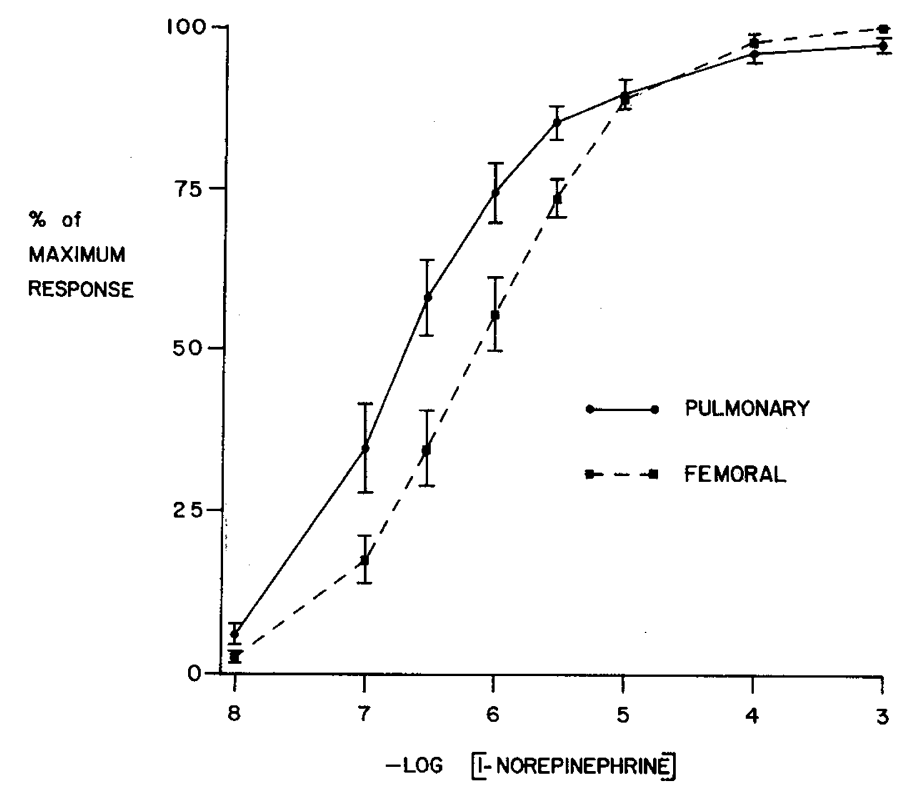

Fig. 3. 1-Norepinephrine (mol/L) concentration-response curves of neonatal ovine intralobar pulmonary $(n=10)$ and femoral $(n=10)$ arteries with uptake inhibition. Bars indicate \pm 1 SEM. Curves different $P<0.05$.

norepinephrine with that of femoral arteries from newborn lambs. When the neuronal and extra-neuronal uptake mechanisms are inhibited, so that the $\alpha_{1}$-receptors are exposed to the same concentration as is present in the remainder of the organ bath, the pulmonary arteries are more sensitive to 1-norepinephrine stimulation than are the femorals. There are a variety of potential mechanisms for this situation including differences in $\alpha_{1}$-receptor sensitivity, distribution, or density, or differences in intracellular biochemical events linking receptor binding to tension development. The present studies cannot address these various possibilities. Similarly, conclusions about potential differences in the entire pulmonary and systemic vasculatures cannot be drawn from isolated vessel studies; however, the present information does confirm the heterogeneity of response to $\alpha$ adrenergic stimulation in various vessels as suggested by Bevan (3) and encourages further investigation in this area.

Our studies of the effect of uptake inhibition were performed as a preliminary step in these experiments. The failure of uptake inhibition to shift the concentration-response curve of the femoral vessels does not rule out the presence of uptake by these vessels. One possible explanation would be that the uptake mechanism may be saturated at a concentration below the threshold of the $\alpha_{1}$-adrenergic receptor in the femoral vessel. Because the pulmonary $\alpha_{1}$-adrenergic receptor may be more sensitive, it could compete with the uninhibited uptake mechanisms for 1-norepinephrine, particularly in the lower concentrations on the concentration-response curve (17). Other possibilities for the apparent differences between the two vessels in uptake could be differences in density or anatomic disposition of adrenergic neuronal uptake sites in the two vessels, undefined effects of $\alpha_{2}$-receptors, or a truly more active endothelial and smoooth muscle uptake in the pulmonary vasculature. The latter possibility is supported by several studies pointing to the importance of endothelial removal of norepinephrine and other vasoactive substances from the circulation $(1,7-9,22,23)$. Conclusions about the physiologic effect of the uptake mechanisms in the newborn period based on these isolated vessel studies remains speculative.

The small difference in the in vitro diameters of the pulmonary and femoral arterial rings resulted from the necessity of avoiding the numerous side branches of the pulmonary vessel when choosing a ring. It is unlikely that the differences demonstrated between in vitro pulmonary and femoral vessel rings can be attributed to 
this small difference in ring diameter because there was no significant correlation between ring diameter and $-\log \mathrm{EC}_{50}$ within each group or between difference in ring diameter and difference in $-\log \mathrm{EC}_{50}$ when comparing pulmonary and femoral arterial rings.

In summary, the present study demonstrates that neonatal ovine intrapulmonary lobar arteries and femoral arteries differ both in their 1-norepinephrine concentration-response relationship and in the effect of inhibition of the uptake mechanisms. These observations suggest a heterogeneity of the response to 1norepinephrine in the neonatal vasculature.

\section{REFERENCES AND NOTES}

1. Alabaster, V. A. and Bakhle, Y. S.: The removal of noradrenaline in the pulmonary circulation of rat isolated lungs. Br. J. Pharmacol., 47: 325 (1973).

2. Besse, J. C. and Furchgott, R. F.: Dissociation constants and relative efficacies of agonists acting on alpha-adrenergic receptors in rabbit aorta. J Pharmacol. Exp. Ther., 197: 66 (1976).

3. Bevan, J. A.: A comparison of the contractile responses of the rabbit basilar and pulmonary arteries to sympathomimetic agonists: further evidence for variation in vascular adrenoceptor characteristics. J. Pharmacol. Exp. Ther., 216: 83 (1981)

4. Cassin, S., Dawes, G. S., and Ross, B. B.: Pulmonary blood flow and vascular resistance in immature foetal lambs. J. Physiol., 171: 80 (1964).

5. Drummond, W. H., Peckham, G. J., and Fox, W. W.: The clinical profile of the newborn with persistent pulmonary hypertension. Clin. Pediatr., 16: 335 (1977).

6. Fox, W. W., Gewitz, M. H., Dinwiddie, R., Drummond, W. H., and Peckham, G. J.: Pulmonary hypertension in the perinatal aspiration syndromes. Pediatrics, 59: 205 (1977)

7. Gewitz, M. H., Pitt, B. R., Laks, H., Hammond, G. L., Talner, N. S., and Gillis, C. N.: Reversible changes in Norepinephrine extraction by the lungs in children with pulmonary hypertension. Pediatr. Pharmacol., 2:517 (1982).

8. Gillespie, J. G. and Muir, T. C.: Species and tissue variation in extraneuronal and neuronal accumulation of noradrenaline. J. Physiol., 206: 591 (1970).

9. Gillis, C.N. and Pitt, B. R.: The fate of circulating amines within the pulmonary circulation. Annu. Rev. Physiol., 44: 269 (1982).

10. Goetzman, B. W. and Milstein, J. M.: Pulmonary vasodilator action of tolazoline. Pediatr. Res., 13: 942 (1979).

11. Goetzman, B. W., Sunshine, P., Johnson, J. D., Wennberg, R. P., Hackel, A., Merten, D. F., Bartoletti, A. L., and Silverman, N. H.: Neonatal hypoxia and pulmonary vasospasm: Response to tolazoline. J. Pediatr., 89: 617 (1976).

12. Greenberg, S., Kadowitz, P. J., Hyman, A., and Curro, F. A.: Adrenergic mechanisms in canine intralobar pulmonary arteries and veins. Am. J. Physiol., 240: H274 (1981).

13. Herlihy, J. T. and Murphy, R. A.: Length-tension relationship of smooth muscle of the hog carotid artery. Circ. Res., 33: 275 (1973).

14. Hessler, J. R. and Cassin, S.: Effects of increased intracranial pressure on pulmonary vascular resistance of fetal and neonatal goats. Am. J. Physiol., 232: H671 (1977).
15. Iverson, L. L.: Role of transmitter uptake mechanisms in synaptic neurotransmission. Br. J. Pharmacol., 41: 571 (1974).

16. Joiner, P. D., Kadowitz, P. J., Hughes, J. P., and Hyman, A. L.: NE and ACh responses of intrapulmonary vessels from dog, swine, sheep, and man. Am. J. Physiol., 228: 1821 (1975).

17. Korones, S. B. and Eyal, F. G.: Successful treatment of "persistent fetal circulation" with tolazoline. Pediatr. Res (abstract), 9:367 (1975).

18. Lagercrantz, H., Bistoletti, P., and Nylund, L.: Sympathoadrenal activity in the foetus during delivery and at birth. In: L. Stern, B. Salle, and B. FriisHansen: Intensive Care in the Newborn. Vol. 3, p. 1 (Masson Publishing, Inc., New York, 1981).

19. Langer, S. Z. and Trendelenburg, U.: The effect of a saturable uptake mechanism on the slopes of dose-respone curves for sympathomimetic amines and the shifts of dose-response curves produced by a competitive antagonist. J. Pharmacol. Exp. Ther., 167: 117 (1969).

20. Lock, J. E., Olley, P. M., and Coceani, F.: Enhanced $\beta$-adrenergic-receptor responsiveness in hypoxic neonatal pulmonary circulation. Am. J. Physiol., 240: H697 (1981)

21. Levin, D. L., Heymann, M. A., Kitterman, J. A., Gregory, G. A., Phibbs, R. H., and Rudolph, A. M.: Persistent pulmonary hypertension of the newborn infant. J. Pediatr., 89: 626 (1976).

22. Masek, K., Svec, P., Dlabac, A., and Raskova, H.: The accumulation of ${ }^{3} \mathrm{H}$ noradrenaline by the lung. Eur. J. Pharmacol., 5: 374 (1969).

23. Nicholas, T. E., Strum, J. M., Angelo, L. S., and Junod, A. F.: Site and mechanism of uptake of ${ }^{3} \mathrm{H}$-1-norepinephrine by isolated perfused rat lungs. Circ. Res., 35: 670 (1974).

24. Padbury, J. F., Roberman, B., Oddie, T. H., Hobel, C. J., and Fisher, D. A.: Fetal catecholamine release in response to labor and delivery. Obstet. Gynecol., 60: 607 (1982).

25. Riemenschneider, T. A. Nielson, H. C. Ruttenberg, H. D., and Jaffee, R. B.: Disturbances of the transitional circulation: Spectrum of pulmonary hypertension and myocardial dysfunction. J. Pediatr., 89: 622 (1976).

26. Siassi, B., Goldberg, S. J., Emmanouilides, G. C., Higashino, S. M., and Lewis, E.: Persistent pulmonary vascular obstruction in newborn infants. J. Pediatr., 78: $610(1971)$

27. Su, C., Bevan, R. D., Duckles, S. P., and Bevan, J. A.: Functional studies of the small pulmonary arteries. Microvasc. Res., 15: 37 (1978).

28. Van Nueten, J.M.: Comparison of isotonic and isometric measurements in isolated arterial tissues. In: J. A. Bevan, T. Godfraind, R. A. Maxwell, and P. M. Vanhoutte: Vascular Neuroeffector Mechanisms, pp. 37-39 (Raven Press, New York, 1980).

29. The authors thank Randy Jerkins and Mildred Jackson for their expert technical help and Rosalind Griffin for secretarial assistance.

30. Requests for reprints should be addressed to: Dr. Robert S. Green, Newborn Center, 853 Jefferson, Room 301, Memphis, TN38163.

31. This work was done during the tenure of an Established Investigatorship from the American Heart Association (CWL) and with funds contributed in part by the Tennessee Affiliate. The research was supported in part by NIH grant HL 22639, a Grant-in-Aid from the American Heart Association with funds contributed in part by the Tennessee Affiliate, a Grant-in-Aid from the Tennessee Lung Association, and by a Biomedical Research Support Grant from The University of Tennessee College of Medicine.

32. Received for publication September 13, 1982.

33. Accepted for publication June 9,1983 